\title{
Free-motion beam propagation factor measurement by means of a liquid crystal spatial light modulator
}

\author{
Jorge Pérez-Vizcaíno, Omel Mendoza-Yero, \\ Raúl Martínez-Cuenca, Lluís Martínez-León, Enrique Tajahuerce \\ and Jesús Lancis
}

GROC, Institute of New Imaging Technologies, Universitat Jaume I, 12071-Castelló, Spain

\begin{abstract}
In contrast to the mechanical scanning procedure described in the standard ISO/DIS 11146, the use of electronically tunable focal length lenses has proved its capability for the measurement of the laser beam propagation factor $\left(\mathrm{M}^{2}\right)$ without moving components. Here, we demonstrate a novel experimental implementation where we use a low-cost programmable liquid crystal spatial light modulator (SLM) for sequentially codifying a set of lenses with different focal lengths. The use of this kind of modulators introduces some benefits such as the possibility for high numerical aperture or local beam control of the phase of the lenses which allows for minimizing systematic errors originated by lens aberrations. The beam width, according to the second order moment of the irradiance, is determined for each focal length by using a digital sensor at a fixed position with respect to the spatial light modulator. After fitting the measured data to the theoretical focusing behavior of a real laser beam, the beam propagation factor is obtained. We successfully validated the results in the laboratory where a full digital control of the measurement procedure without mechanical scanning was demonstrated.
\end{abstract}

Index Terms-Beam propagation factor, laser, spatial light modulators.

\section{INTRODUCTION}

After 50 years from the invention of the laser, this light source plays a major role in several areas of current importance such as optical communications, health care and industrial applications. Most of the above applications require a detailed characterization of the laser beam profile. A monochromatic Gaussian laser beam $\left(\mathrm{TEM}_{00}\right)$ is defined through the

Manuscript received July 26, 2006. This research was funded by the Dirección General de Investigación Científica y Técnica, Spain, under the project FIS2004-02404 and FEDER. We also acknowledge financial support from the Generalitat Valenciana (grant GV05/110).

Jorge Pérez-Vizcaíno, Omel Mendoza-Yero, Raúl Martínez-Cuenca, Lluís Martínez-León, Enrique Tajahuerce and Jesús Lancis are with the Departament de Ciències Experimentals, Universitat Jaume I, 12080 Castelló, Spain (e-mail: lancis@exp.uji.es). propagation direction, the peak amplitude, the waist location and one additional parameter (the Rayleigh range $z_{R}$, the waist radius $\sigma_{\omega}$ or the beam divergence $\theta$ ) [1]. For real lasers, the so-called $M^{2}$ propagation factor provides a measurement of the deviation of the beam with respect to that of a $\mathrm{TEM}_{00}$ Gaussian beam due to contributions from higherorder transverse modes, truncation or other distortions introduced by lenses, apertures, or other optic systems. The determination of the beam propagation factor is crucial for both scientific and industrial fields. As a matter of fact, the efficiency of multiphoton processes originated through tight focusing of laser beams depends on the powers of the irradiance distribution, so having a small $M^{2}$ means having a better product yield. Also, in the industry field, the measurement of $M^{2}$ is meaningful for applications such as hole drilling where a beam with a $M^{2}$ factor of two will produce a hole twice bigger than the expected for a $\mathrm{TEM}_{00}$ Gaussian beam.

The ISO standard (ISO/DIS 11146) provides a convenient method for $M^{2}$ measurement. In this technique, the laser beam to be evaluated is relayed through a lens in a fixed position and multiple beam-width measurements are made along the waist region behind the lens [2]. Finally the beam propagation factor is obtained through the fitting of the experimental data. The beam width is measured in agreement with the second-order moment criterion. This method involves mechanical scanning of the intensity sensor along the beam propagation direction. In practical terms, this requires a high degree of alignment and parallelism. Also, the measurement is time-consuming (of the order of tens of seconds) and thus, sensible, to environmental fluctuations and beam changes.

Fast and easily applicable methods are needed for practical use due to ever more stringent requirements regarding the quality of laser beams. Very recently, faster and free-motion methods have been proposed. Among them, we mention those based on the use of a Shack-Hartmann wavefront sensor that provides unprecedented temporal resolution (up to $10 \mathrm{kHz}$ ) $[3,4]$ and those based on simultaneous capture of the waist at several Rayleigh ranges allowing the instantaneous fit of the $M^{2}$ curve at video rate [5]. On the other hand, it has also been proposed the idea of measuring the $M^{2}$ beam parameter using 
an electronically controlled variable focus lens [6-8]. To the best of our knowledge, up to present, all of the experimental implementations are limited to a variable-focus electrowetting-driven liquid lens in conjunction with an alldigital micromirror device [6-8]. In contrast to conventional focusing systems, such lenses suffer from a relatively low pupil diameter, and subsequent short focal length, which in some circumstances prevents its applicability in practical laser environments [9]. More interestingly, some voltage-dependent beam aberrations (particularly spherical, comma, and chromatic aberrations) are intrinsic to varioptic liquid lenses that, in this way, introduce some amount of systematic error in $M^{2}$ measurement [10]. More interestingly, conventional liquid lenses suffer from a strong astigmatism, distortion or light scattering during focus change that need to be corrected for by using sophisticated electrode design.

In this paper, following the idea originally proposed by the Riza group in [6], electronically controlled variable focal length lenses are codified onto a phase-only liquid crystal spatial light modulators (SLMs) for beam parameter measurement. In our method, a set of lenses with different focal lengths is codified onto the SLM by quadratic phase modulation and the diffracted beam width, according to the second order moment of the irradiance distribution, is determined for each focal length by using a CCD at a fixed position. The use of this kind of modulators introduces some benefits such as the possibility for a bigger numerical aperture or local beam control of the phase of the lenses which allows for minimizing systematic errors originated by the lens aberrations in the standard procedure. Yet, our technique also opens the possibility of real-time fine-tuning of laser beams through the programmable nature of phase-only SLMs.

Liquid-crystal displays working as electronically addressed SLMs have been widely used to generate programmable diffractive lenses [11] and the mathematical models to encode the lens function in a device constrained by its pixilated structure and phase quantization analyzed [12]. In this paper, we also carry out the analysis of the artifacts (such as multiple focal lengths and higher-order diffractions) due to the pixilated and the quantized nature of the lenses codified onto the SLM in beam propagation factor measurements. This analysis is carried out by means of a computer simulation. Finally, SLMs permit programmable lenses working under broadband or white-light illumination that allow to extend the proposed technique for different wavelength ranges without the need of previous calibration [13,14]. Apart from lens codification, the use of phase-only SLMs in different tasks for optical information processing and adaptive optics has been extensively reported since the 90ths with extensive success [15-17].

\section{EXPERIMENTAL SETUP AND THEORY}

\section{A. Beam characterization}

Following the ISO standard (ISO11146), for a properly aligned astigmatic beam propagating along the $z$ axis, the second-order spatial moments are used to describe the beam diameter and the orientation of the irradiance distribution at a given transverse plane. For instance, for the horizontal
Cartesian coordinate $x$, we have for the root-mean-square (rms) width $\sigma_{x}^{2}(z)=\left\langle x^{2}\right\rangle_{z}-\langle x\rangle_{z}^{2}$, where

$$
\left\langle x^{n}\right\rangle_{z}=\frac{1}{I_{o}} \int_{-\infty}^{+\infty} x^{n}|\psi(x, y, z)|^{2} d x d y
$$

is the normalized n-order moment of the beam intensity $|\psi(x, y, z)|^{2}$. In the above equation $I_{o}$ denotes the 0 -order moment of the function $\mid \psi(x, y, z)^{2}$. Likewise, an analogous definition holds for the vertical coordinate $y$. For a TEM ${ }_{00}$ Gaussian beam, the rms width propagates along the axial coordinate in accordance with the simple quadratic relationship

$$
\sigma_{x}^{2}(z)=\sigma_{w x}^{2}\left\lfloor 1+\left(\frac{z-z_{w x}}{z_{R x}}\right)^{2}\right\rfloor,
$$

where $\sigma_{w x}$ is the rms width at the waist plane $z=z_{w x}$ and $z_{R x}=4 \pi \sigma_{w x}^{2} / \lambda$ is the Rayleigh range of the monochromatic beam of wavelength $\lambda$. Also, the second-order angular moments are the pendants of the second-order spatial moments in the far field of the beam giving the beam divergence

$$
\theta_{\sigma_{x}}=4 \lim _{z \rightarrow \infty} \frac{\sigma_{x}}{z}
$$

The $M^{2}$ propagation factor for a simple astigmatic beam is defined through the product of the beam waist width and the divergence at far field [1]

$$
M_{x}^{2}=\frac{\pi}{\lambda} \sigma_{w x} \theta_{\sigma_{x}}
$$

and

$$
M_{y}^{2}=\frac{\pi}{\lambda} \sigma_{w y} \theta_{\sigma_{y}},
$$

in horizontal and vertical directions, respectively. As is well known, the value of $M^{2}$ for a $\mathrm{TEM}_{00}$ Gaussian beam is 1 . It is not possible to find a lower value of the beam propagation factor for actual realizable beams. The Rayleigh range is reduced accordingly as $z_{R x}=4 \pi \sigma_{u x}^{2} / M_{x}^{2} \lambda$ [1]. This parameter accounts for the spreading of the beam width in accordance with Eq. (2) with a reduced Rayleigh range and provides a measure of the departure from the Gaussian behavior. Also, $M^{2}$ is invariant through propagation along ABCD optical systems. From a practical point of view, a beam with a certain width showing lower $M^{2}$ value will behave better for focalization (collimation) purposes in the sense that 
the minimum spot size (minimum beam divergence) for a given optical system will be smaller.

\section{B. Beam propagation in lens-like media}

For future interest, we enter into detail about beam transformation through a very simple ABCD system consisting of a single lens of focal length $f$ and two portions of free space (see Fig.1 for notation). We are interested in how the changes in the focal length affect to beam parameters after the lens and how to express these changes in terms of the input beam parameters. By geometrical transformations, it is straightforward to obtain the formulas that relate the parameters of the incident beam to those of the beam that emerges from the lens (with "s" subscript) $[1,18]$. In this way, we find for both the horizontal and the vertical directions

$$
\begin{aligned}
& z_{w s}=f-\beta\left(z_{w}+f\right), \\
& \sigma_{w s}^{2}=\beta \sigma_{w}^{2},
\end{aligned}
$$

and

$$
z_{R s}=\beta z_{R}
$$

where the magnification parameter $\beta$ is defined as

$$
\beta=\left(1+\frac{2 z_{w}}{f}+\frac{z_{w}^{2}+z_{R}^{2}}{f^{2}}\right)^{-1} .
$$

By taking into account Eqs.(2), (7) and (8), we find the following propagation law for the squared rms width at any transversal plane located at a distance $z$ behind the lens [18]

$\sigma_{s}^{2}(z)=\frac{M^{2} \lambda}{4 \pi z_{R}}\left\lfloor z_{w}^{2}+z_{R}^{2}+z^{2}-2 z z_{w}+2 \frac{z^{2} z_{w}-z\left(z_{w}^{2}+z_{R}^{2}\right)}{f}+\frac{z^{2}\left(z_{w}^{2}+z_{R}^{2}\right)}{f^{2}}\right\rfloor$

This equation holds for both the horizontal and the vertical directions. Eq.(10) can be recognized as a simple algebraic expression in terms of the focal length of the SLM

$$
\sigma_{s}^{2}=a+\frac{b}{f}+\frac{c}{f^{2}}
$$

where the fitting parameters can be easily related to the actual beam parameters of the incident beam through

$$
\begin{aligned}
& M^{2}=\frac{4 \pi}{\lambda z^{2}} \sqrt{a c-\frac{b^{2}}{4}}, \\
& z_{w}=\frac{\frac{c}{z}+\frac{b}{2}}{a+\frac{1}{z}\left(\frac{c}{z}+b\right)},
\end{aligned}
$$

and

$$
z_{R}^{2}=\frac{c}{a+\frac{1}{z}\left(\frac{c}{z}+b\right)}-\left[\frac{\frac{c}{z}+\frac{b}{2}}{a+\frac{1}{z}\left(\frac{c}{z}+b\right)}\right]^{2} .
$$

Equation (11) states that by transforming the beam with several spherical lenses of different focal length, the input beam parameters can be determined from a least squares fit, in accordance with Eqs.(12), (13), and (14), if we evaluate the corresponding rms width for each beam profile by using a digital camera at a fixed axial location. This is the underlying idea of our free-motion full digital technique for beam propagation measurement.

\section{SPATIAL LIGHT MODULATOR CALIBRATION}

In our approach, a set of lenses with tunable focal length is codified sequentially into a phase-only liquid crystal SLM. A variable quadratic-phase factor in the spatial domain displayed on the SLM plays the role of the programmable lens. Whenever the focal length is changed, a different axial plane is imaged onto the beam profiler, which is kept at a fixed position (see Fig. 2). In this way, a complete digital control of the measurement system is attained without the need of any moving component as a main difference with the ISO standard procedure.

We used a reflective Liquid Crystal on Silicon (LCoS) SLM, Holoeye Pluto, with a panel size of 0.7 ", $1920 \times 1080$ pixels, a fill factor of $87 \%$ and a pixel pitch of $8 \mu \mathrm{m}$. This device achieves a phase modulation exceeding $2 \pi$ rad for the working wavelength in the visible region and near IR. The SLM is based on a nematic-electronic controlled birefringence mode, in which the liquid crystal molecules are initially parallel to the electrodes but tend to tilt following the direction of the applied electric field. The amplitude and polarization modulations are assumed to be negligible. Pixels are individually addressed by sending gray-level images to the modulator. Thus, every gray-level $g$ corresponds to a given phase shift. The display has an 8-bit controller that provides 256 different values of $g$. The operation curve of the modulator shows in general a non-linear behavior, so a careful calibration is required.

The SLM has been characterized in detail in our laboratory. We calibrated the SLM for the operating wavelength of the laser beam at $633 \mathrm{~nm}$ through the measurement of the intensity modulation in a polarimetric setup (Fig.3) as a function of the addressing gray level. The input linear polarization was set at $45^{\circ}$ relative to the axis in which liquid crystal molecules are aligned. The analyzer was set at the same orientation as the input polarization. This configuration is termed as the "intensity modulation mode" as phase variations are converted onto intensity fluctuations for calibration purposes [19,20]. Different gray-level images are sent to the SLM and the optical transmittance defined as the ratio between the output and the input light intensity is found to be 


$$
T(g)=\frac{1}{2} \cos (\phi)
$$

with $\phi$ the phase shift. In this way, the calibration curve $\phi=f(g)$ is theoretically expressed through

$$
\phi=\cos ^{-1}[2 T(g)]
$$

Fig. 4 shows the calibration curve for $633 \mathrm{~nm}$, in which just a limited range of gray levels, chosen from a convenient interval among the $[0,255]$ scale, is needed to get the required $2 \pi$ phase modulation.

From a practical point of view, energy limitations arise from the use of the SLM. Although a neutral density filter can be used to attenuate the laser intensity, it is convenient to recall that the laser beam power incident onto the SLM should remain under the damage threshold of the display. For the case of continuous wave laser operation, this quantity is in the order of several $\mathrm{W} / \mathrm{cm}^{2}$ for a laser wavelength in the visible region of the spectrum and near IR. It should be noted that this quantity quickly decreases for UV radiation that prevents the technique to operate at this spectral region.

\section{ANALYSIS OF THE LENS CODIFICATION ONTO THE SPATIAL LIGHT MODULATOR}

With the previous result we sent to the modulator the gray levels corresponding to the quadratic phase imparted by a lens of focal length $f$; i.e, $\phi(r)=k r^{2} / 2 f$, with $k$ the wave number and $r$ the radial coordinate onto the SLM pupil. In practice the SLM displays the phase wrapped in $2 \pi$ steps [14] (see Fig. 5). Note that this wrapping may lead to aliasing in the outer regions of the lens since the width of each wrapped zone decreases with the distance to the center. A detailed mathematical model that describes the behavior of lenses encoded in a low-resolution device can be found in Ref. [12]. In short, Carcolé et al demonstrated the existence of an optimum focal length for best lens performance in terms of the FWHM focal distribution for a given codification device. The point is to define blocks of pixels that always contribute constructively to the focal intensity. For current pixel size in the $\mu \mathrm{m}$ range, the FWHM spreading at the focal plane is remarkably low for focal lengths extending from a few millimeters to several centimeters. In a simplified model, the focal length of the codified lens consisting of several Fresnel zones is directly related to the outermost zone width, $\Delta r$, and the lens diameter, $d$, through $f=d \Delta r / \lambda$. In practice, the outermost zone must be displayed with at least two pixels to avoid aliasing, so $\Delta r$ actually equals the pixel size. In addition, the lens diameter should be adjusted to several times the beam width over the SLM to avoid clipping of the laser beam. For our experiment, this leads to a minimum focal length of 25 $\mathrm{mm}$. There is not a clear criterion to establish the maximum focal length that can be codified since it depends on the required lens efficiency. For the experiment the maximum focal length $(f \cong 80 \mathrm{~cm})$ is considered to provide accurate results. It is also worth mentioning that some care must be paid to block out light diffracted into the higher diffraction orders of the pixilated diffractive lens codified onto the SLM.
We conducted some computer simulations in order to evaluate the influence of pixilation and quantization of the SLM in beam propagation factor measurements. For the analysis, we considered a $\mathrm{TEM}_{00}$ Gaussian beam, with the generalization to more complex beam profiles automatic. The simulation was carried out in the following way. First, the beam rms width was analytically evaluated at the sensor plane assuming a non-pixilated, non-quantized lens profile. Afterwards, the effect of the sampled and quantized Fresnel lens onto the $\mathrm{TEM}_{00}$ Gaussian beam was simulated in the computer using the Fresnel transform as a valid diffraction model. The pixel size was chosen as a parameter. The number of pixels in the SLM (the lens pupil) was chosen to cover the full beam diameter so that no clipping artifacts were introduced. In this way, the rms width of the transformed beam was calculated using computer data for the simulated irradiance distribution at the sensor plane and the results compared to the analytical values. It is worthwhile to mention that in order to evaluate the simulated rms width, the irradiance distribution was clipped and zoomed around the central maxima of the diffractive orders generated by the pixilated structure of the SLM. For the simulation we chose a wavelength of $632 \mathrm{~nm}$, a rms width of $0.25 \mathrm{~mm}$ at the SLM plane, a lens pupil of $4.8 \mathrm{~mm}$, and the sensor plane was located $43.5 \mathrm{~cm}$ away from the SLM, which correspond to typical values for the optical implementation in the next section. Pixel size at the output plane was kept fixed to $8 \mu \mathrm{m}$ which corresponds roughly to the pixel size of the CCD in the experimental section. Three different values for the focal length codified onto the SLM were considered which correspond to the extreme lenses of $100 \mathrm{~mm}$ and $700 \mathrm{~mm}$ and the intermediate value of $150 \mathrm{~mm}$.

The results for the beam rms width at the sensor plane in terms of the pixel size of the SLM are shown in Fig. 6. Analytical values are labelled with a cross, whereas simulated values are shown as a circle. Also, we plot in Fig.7 the irradiance distribution around the central maxima for the lens with focal length $100 \mathrm{~mm}$ and a pixel size of $1 \mu \mathrm{m}$ and $64 \mu \mathrm{m}$. In all cases, a pixel size lower than $10 \mu \mathrm{m}$ leads to an excellent agreement between simulated and analytical values, which confirm that sampling and quantization effects are negligible for a pixel sizes 25 times lower than the beam width. However, we report strong discrepancies for lenses codified onto low-resolution SLMs. These artifacts are more significant in the low focal length regime, even with relative errors as high as $400 \%$. This fact makes it impossible the use of our technique in this regime.

In terms of the speed of operation, the system supports continuous signal acquisition for video rates up to $60 \mathrm{~Hz}$ which, in practical conditions, determines the bottleneck in terms of measurement speed for the beam propagation factor. From a spectral point of view, liquid crystal SLMs can be operated under broadband illumination although, for this case, severe chromatic aberrations coming from refractive index chromatism, the diffraction chromatism, and the quantization chromatism are expected $[13,14]$. Although, for narrowband laser beams working at different spectral regions the SLM the system should be, in principle, recalibrated, even for this 
situation the chromatic compensation for a set of discrete wavelengths can alleviate the above matter [13,14].

\section{EXPERIMENTAL RESULTS}

According with the above procedure, we measured the beam propagation factor of a commercial He-Ne laser $(\lambda=632 \mathrm{~nm}$, $20 \mathrm{~mW}$ ) emitting a $\mathrm{TEM}_{00}$ mode with linear polarization as a coherent beam source and beam diameter of about $2 \mathrm{~mm}$. It was characterized employing both the standard ISO approach and our procedure. In both cases, beam profiling was performed by means of a 14bit digital charge-coupled device camera, 1360x1024 pixels, size $6.45 \mu \mathrm{m}$ (WinCamD-UCD23) previously calibrated for the measurement of the laser beam parameters. Camera data processing was performed using commercial software. The data for the squared beam radius are plotted in Fig.8 (x axis) and Fig.9 (y axis) against the focal length displayed onto the SLM. The beam profiler was fixed at a distance of $43.5 \mathrm{~cm}$ from the SLM. We oversampled the focal length range between $10 \mathrm{~cm}$ and $80 \mathrm{~cm}$ with 140 sampling points. Focal lengths variations of a few millimeters generate focal distributions with a detectable focal depth in terms of the CCD pixel size. In Fig.10 a picture of the focal distributions for two focal lengths differing in $5 \mathrm{~mm}$ is shown. Note that the ISO method only requires ten measurement points along the axis of propagation (five around the waist and five two Rayleigh ranges distal to the test lens). A hyperbolic fit yields $M^{2}$ values of $1.12 / 1.15$ in horizontal/vertical direction, respectively. The effort for a complete measure (140 sampling points) lasted around 10s. This time interval is shortened if the number of sampling points is reduced according to the ISO standard. Also, the measurement interval can be further reduced if the software control is implemented onto the graphic processing unit of the computer. The results for the beam propagation factor following the ISO approach were $1.07 / 1.08$ in horizontal/vertical directions after mechanical scanning of the beam profiler along the propagation direction. Thus a concordance level of less than $5 \%$ between both methods is found. Yet, the accuracy of both methods is comparable and mainly determined by beam profiling measurement accuracy. Using a 14-bit beam profiler, beams diameters are obtained with an accuracy better than $2 \%$ that translates to better than $4 \%$ in $M^{2}$ determination.

\section{CONCLUSIONS}

To the best of our knowledge, we have experimentally validated the first beam propagation factor analyzer based on a liquid crystal SLM and a beam profiler using the idea of electronically controlled focal length lenses proposed originally by Riza et al [6]. By using a digital sensor at a fixed position, we measure the beam width for each focal length that is codified onto the SLM. This analyzer provides full digital control, obtaining a compact and quick measurement method free from mechanical scanning. After fitting the measured data to the theoretical focusing behavior of a real laser beam, the beam propagation factor is obtained. The method has been experimentally performed in the laboratory and has demonstrated comparable performance to that of the ISO approach (within a concordance level of less than 5\%) but free from artefacts associated to moving components.

\section{ACKNOWLEDGMENT}

This research was funded by Ministerio de Ciencia e Innovación, Spain, under the project FIS2010-15746 and FEDER. Also financial support from the agreement between Universitat Jaume I and Fundación Caixa Castelló (project P11B2009-36) is acknowledged.

\section{REFERENCES}

[1] B.E.A. Saleh and M.C. Teich. Fundamentals of photonics, Second edition, Bahaa E.A. Saleh Series editor.,p.79-87 (1989).

[2] Test method for laser beam parameters: Beam width, divergence angle and beam propagation factor, Document ISO/DIS 11146, International Organization for Standardization, 1996.

[3] B. Schäfer, M. Lübbecke, and K. Mann, "Hartmann-Shack wave front measurements for real time determination of laser beam propagation parameters", Rev. Sci. Ins. 77, 053103 (2006).

[4] J.V.Sheldakova, A.V.Kudryashov, V.Y.Zavalova, and T.Y.Cherezova, "Beam quality measurements with ShackHartmann wavefront sensor and M2-sensor: comparison of two methods", Proceedings of SPIE 4493, 285-293 (2002).

[5] A. M. Cary, J. L. Guttman, R. Chirita, and D. W. Peterman, "Instantaneous measurement of beam propagation ratio in realtime", Proceedings of SPIE 6871, 687103-687103-11 (2008).

[6] Mumtaz Sheikh and Nabeel A. Riza, "Motion-free hybrid design laser beam propagation analyzer using a digital micromirror device and a variable focus liquid lens", Appl. Opt. 49, D11-D16 (2010).

[7] M. Sheikh, P. J. Marraccini and, N. A. Riza, "Laser beam characterization using agile digital-analog photonics", Proceedings of SPIE 7675, 767508 (2010).

[8] P. J. Marraccini and, N. A. Riza, "Multimode laser beam characterization using agile digital-analog photonics", Proceedings of SPIE 8026, 8026OE (2010)

[9] S. Kuiper and B.H.W. Hendriks, "Variable-focus liquid lens for miniature cameras", Appl. Phys. Lett. 85, 1128 (2004).

[10] H. Ren and S. T. Wu, "Variable-focus liquid lens", Opt. Express 15, 5931-5936 (2007)

[11] V. Laude, "Twisted-nematic liquid-crystal pixilated active lens", Opt. Commun. 153, 134-152 (1998).

[12] E. Carcolé, J. Campos, and S. Bosch, "Diffraction theory of Fresnel lenses encoded in low-resolution devices", App. Opt. 33, 162-174 (1994).

[13] A. Márquez, C Iemmi, J. Campos, and M. J. Yzuel, "Achromatic diffractive lens written onto a liquid crystal display", Opt. Lett. 31, 392-392 (2006)

[14] M. S. Millán, J. Otón, and E. Pérez-Cabre, "Dynamic compensation of chromatic aberration in a programmable diffractive lens", Opt. Express 14, 6226-6242 (2006).

[15] J. L. Martinez, I. Moreno, and E. Ahouzi, "Diffraction and signal processing with a liquid crystal microdisplay", Eur. J. Phys. 27, 1221-1231 (2006).

[16] C. Maurer, A. Jesacher, S. Bernet, and M. Ritsch-Marte, "What spatial light modulators can do for optical microscopy", Laser Photon. Rev. 5, 81-101 (2011).

[17] J. Arines, V. Durán, Z. Jaroszewicz, J. Ares, E. Tajahuerce, P. Prado, J. Lancis, S. Bara, and V. Climent, "Measurement and compensation of optical aberrations using a single spatial light modulator", Opt. Express 15, 15287-15292 (2007).

[18] L. Martí-López, O. Mendoza-Yero, and J. A. Ramos-de-Campos, "Propagation of polychromatic Gaussian beams through thin lenses," J. Opt. Soc. Am. A 18, 1348-1356 (2001). 
[19] F. Li, N. Mukohzaka, N. Yoshida, Y. Igasaki, H. Toyoda, T. Inoue, Y. Kobayashi, and T. Hara, "Phase Modulation Characteristics Analysis of Optically-Addressed Parallel-Aligned Nematic Liquid Crystal Phase-Only Spatial Light Modulator Combined with a Liquid Crystal Display", Opt. Rev. 5, 174-178 (1998).

[20] Ll. Martínez-León, P. Clemente, E. Tajahuerce, G. Mínguez-Vega, O. Mendoza-Yero, M. Fernández-Alonso, J. Lancis, V. Climent, and P. Andrés, "Spatial-chirp compensation in dynamical holograms reconstructed with ultrafast lasers", Appl. Phys. Lett. 94, 011104 (2009).

First A. Author (M'76-SM'81-F'87) and the other authors may include biographies at the end of regular papers. Biographies are often not included in conference-related papers. This author became a Member (M) of IEEE in 1976, a Senior Member (SM) in 1981, and a Fellow (F) in 1987. The first paragraph may contain a place and/or date of birth (list place, then date). Next, the author's educational background is listed. The degrees should be listed with type of degree in what field, which institution, city, state, and country, and year degree was earned. The author's major field of study should be lower-cased.

The second paragraph uses the pronoun of the person (he or she) and not the author's last name. It lists military and work experience, including summer and fellowship jobs. Job titles are capitalized. The current job must have a location; previous positions may be listed without one. Information concerning previous publications may be included. Try not to list more than three books or published articles. The format for listing publishers of a book within the biography is: title of book (city, state: publisher name, year) similar to a reference. Current and previous research interests end the paragraph.

The third paragraph begins with the author's title and last name (e.g., Dr. Smith, Prof. Jones, Mr. Kajor, Ms. Hunter). List any memberships in professional societies other than the IEEE. Finally, list any awards and work for IEEE committees and publications. If a photograph is provided, the biography will be indented around it. The photograph is placed at the top left of the biography. Personal hobbies will be deleted from the biography. 


\section{List of figures}

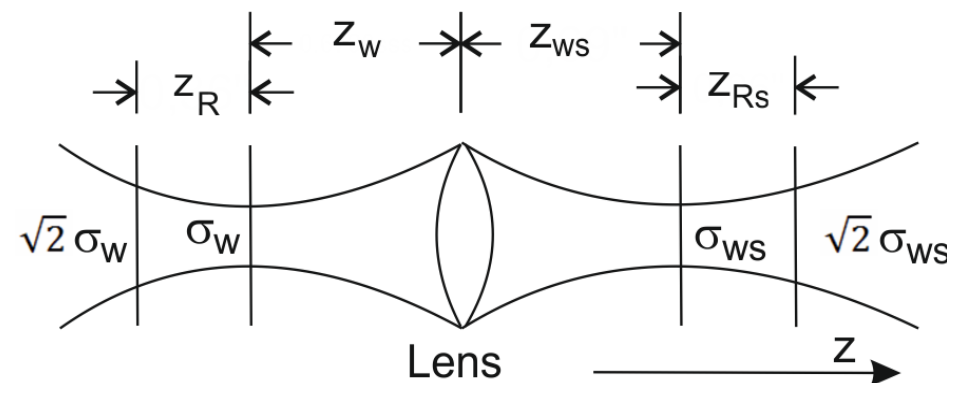

Figure 1. Beam transformation by a thin lens. $Z_{W S}$ and $Z_{W}$ are measured from the lens to the waist of the beam before the lens and behind the lens. $Z_{W S}>0$ for $Z_{w S}$ behind the lens and $z_{w}>0$ for $Z_{w}$ located before the lens. 


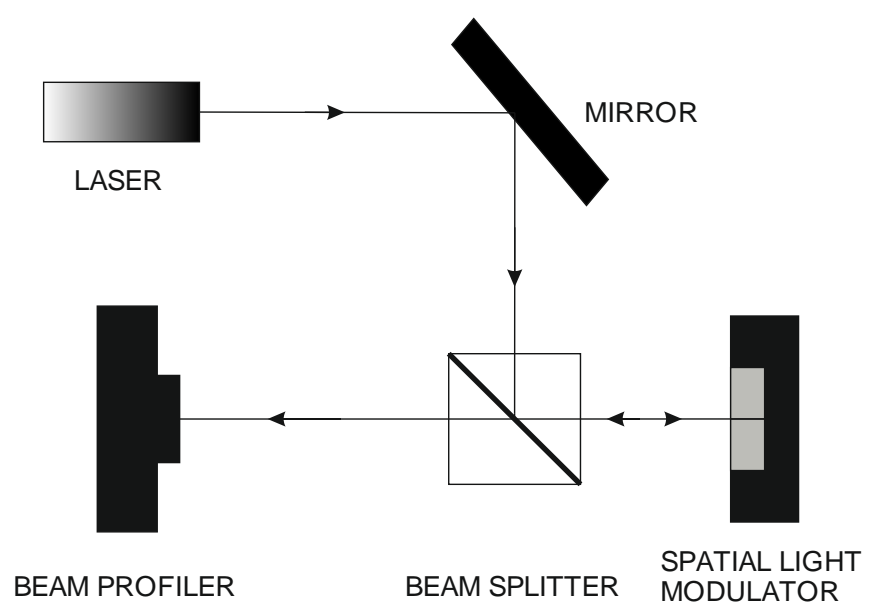

Figure 2. Schematic of the optical device 


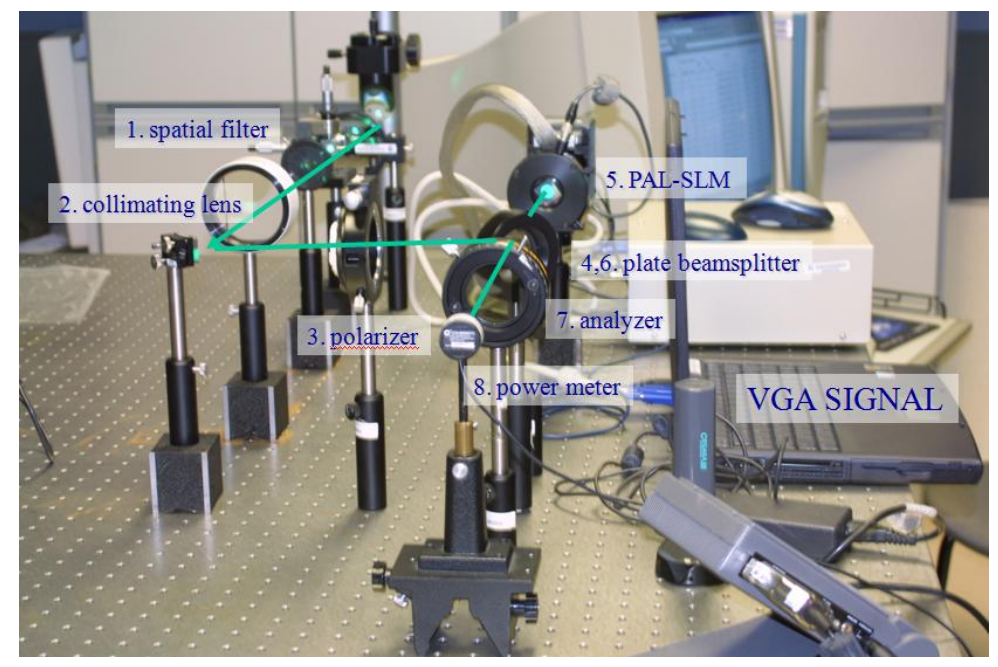

Figure 3. Polarimetric set-up for SLM calibration 


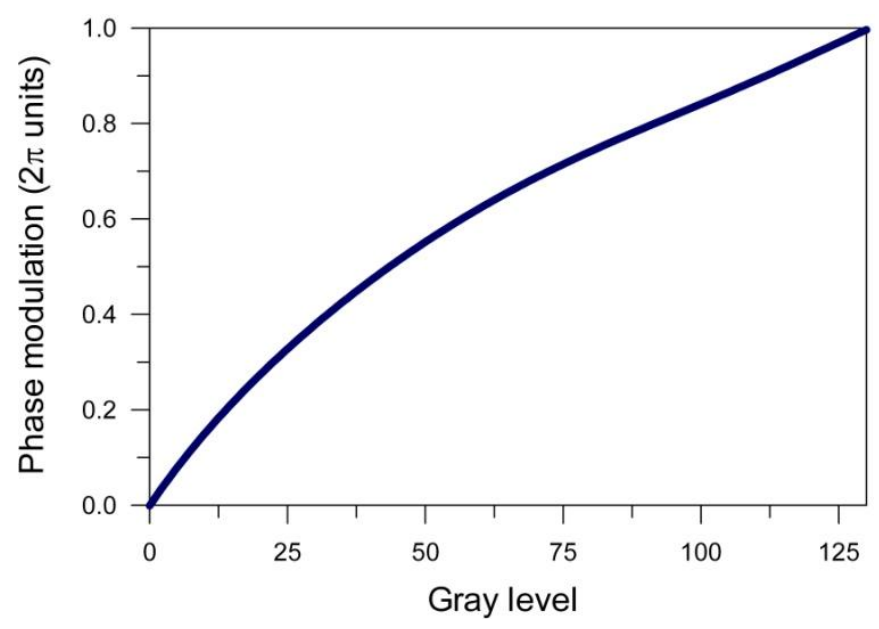

Figure 4. Phase modulation versus gray level. It is not necessary to use the full 256 gray level scale to achieve a $2 \pi$ phase modulation. 


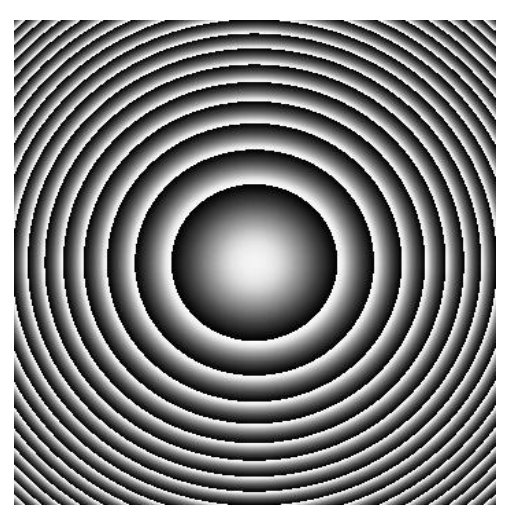

Figure 5. Phase map corresponding to a diffractive lens. The fringes are concentric rings with increasing phase from 0 to $2 \pi$. Fringes width decrease with distance to the center what can produce aliasing. 

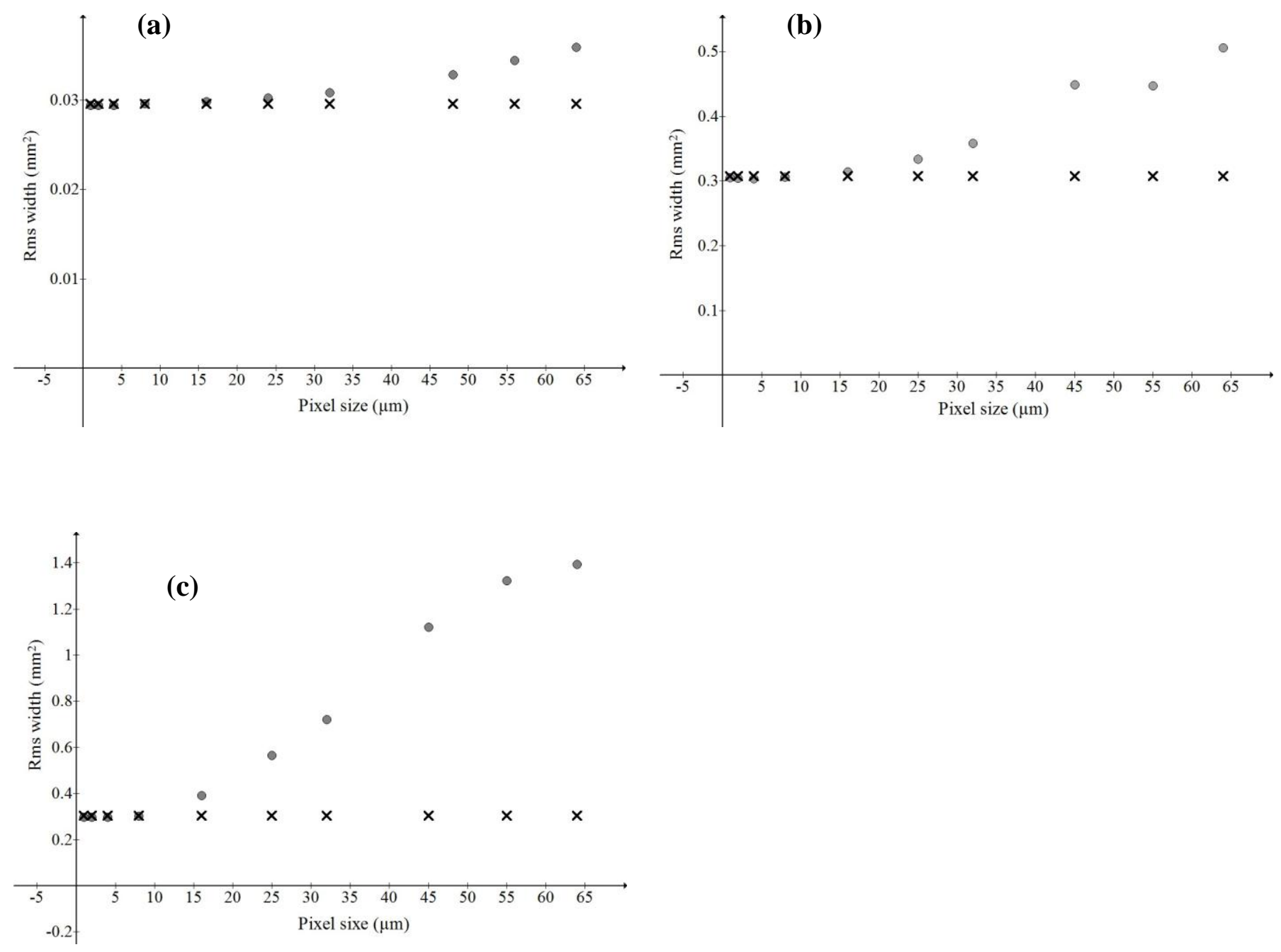

Figure 6. Plot of the beam rms width at the sensor plane in terms of the pixel size of the SLM for a codified focal length of 700 $\mathrm{mm}$ (a), $150 \mathrm{~mm}$ (b), and $100 \mathrm{~mm}$ (c). Analytical values are labelled with a cross, whereas simulated values are shown as a circle. 

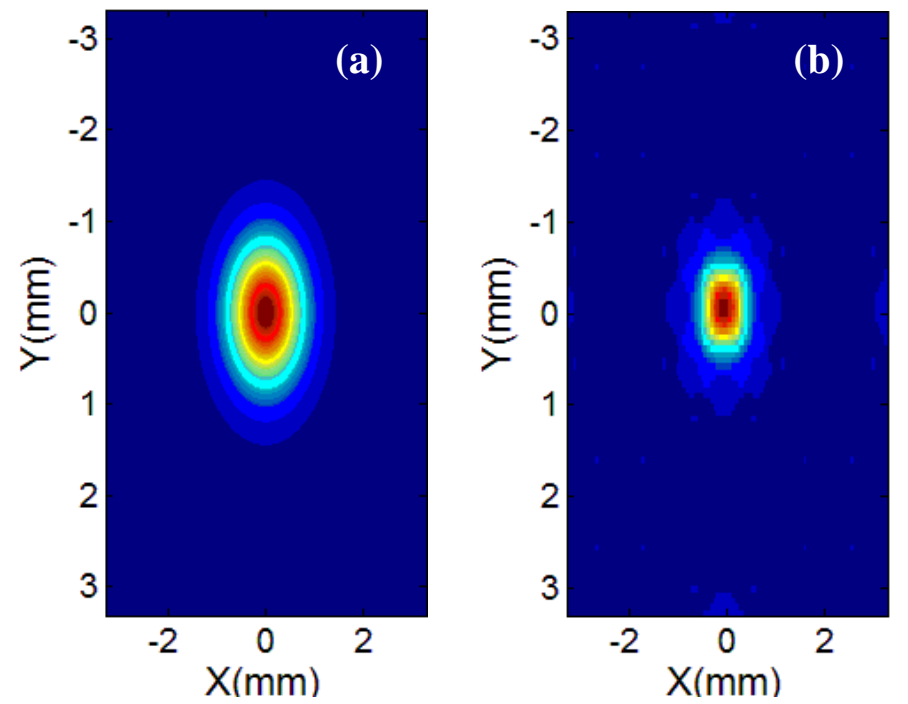

Figure 7. Simulated irradiance distribution around the central maxima for a lens with focal length $100 \mathrm{~mm}$ codified onto an SLM with a pixel size of $1 \mathrm{~mm}(\mathrm{a})$; and $64 \mathrm{~mm}(\mathrm{~b})$. 


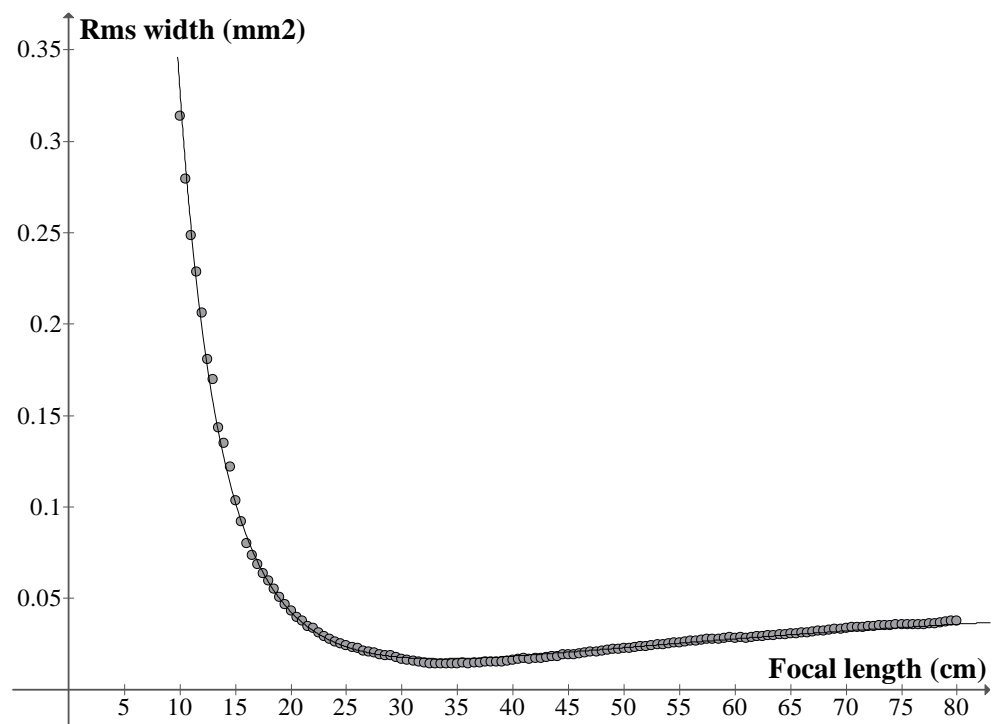

Figure 8. Experimental results for $\mathrm{x}$ axis 


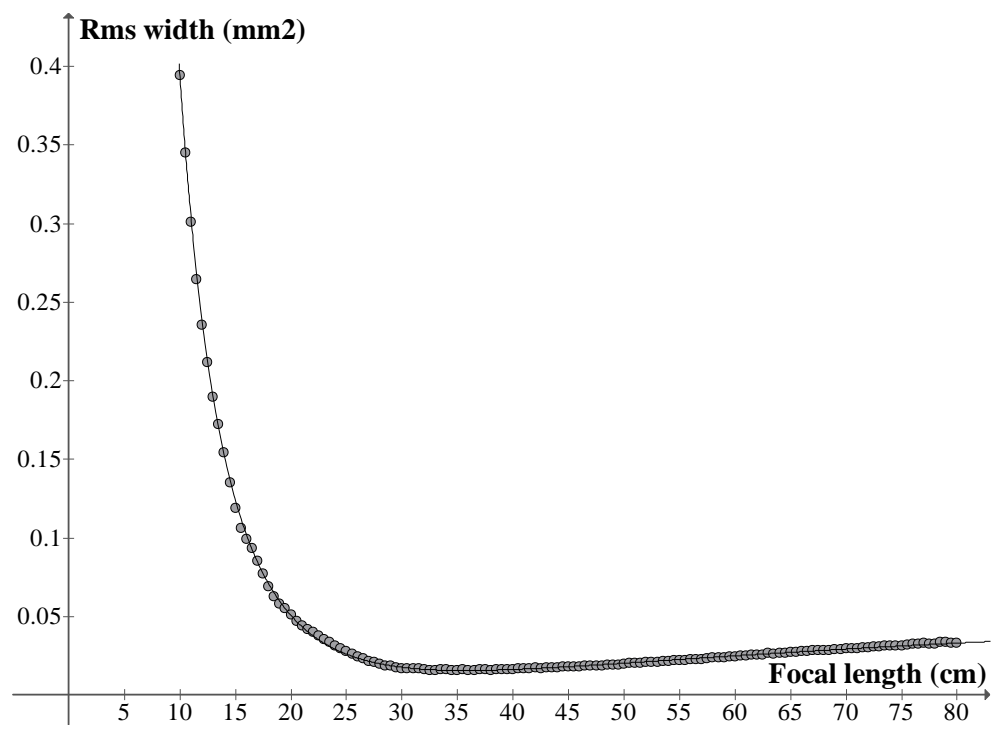

Figure 9. Experimental results for y axis 

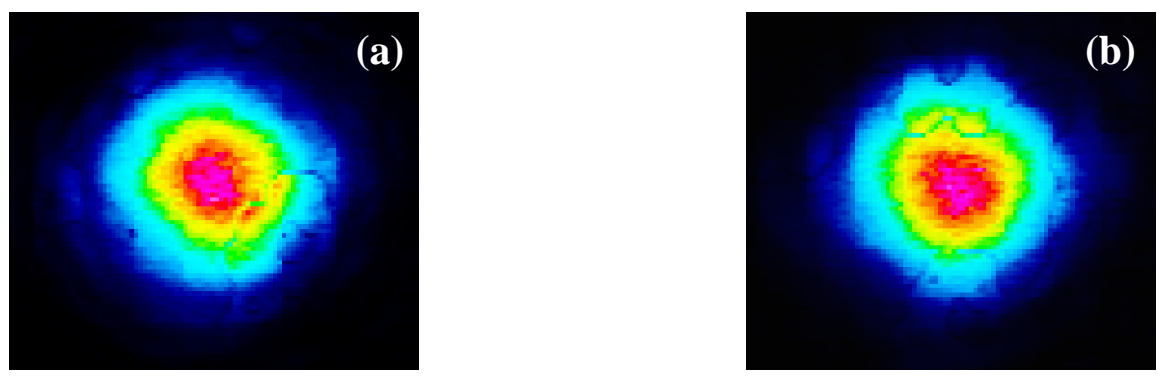

Figure 10. Experimental irradiance distributions at the sensor plane when the codified focal length at the SLM are $22.5 \mathrm{~cm}$ (a) and $23 \mathrm{~cm}(\mathrm{~b})$. 